\title{
Blockade of TRPA1 with HC-030031 attenuates visceral nociception by a mechanism independent of inflammatory resident cells, nitric oxide and the opioid system
}

\author{
L.M.S. Pereira1, R.C.P. Lima-Júnior ${ }^{1}$, A.X.C. Bem¹, C.G. Teixeira ${ }^{1}$, L.S. Grassi ${ }^{1}$, R.P. Medeiros ${ }^{1}$, \\ R.D. Marques-Neto ${ }^{1}$, R.B. Callado ${ }^{1}$, K.S. Aragão ${ }^{1}$, D.V.T. Wong ${ }^{1}$, M.L. Vale ${ }^{1}$, G.A.C. Brito ${ }^{2}$, R.A. Ribeiro ${ }^{1}$ \\ 1 Department of Physiology and Pharmacology, Faculty of Medicine, Federal University of Ceará, Fortaleza, Ceará, Brazil \\ 2 Department of Morphology, Faculty of Medicine, Federal University of Ceará, Fortaleza, Ceará, Brazil
}

\section{Correspondence \\ Roberto César Pereira Lima Júnior \\ E-mail: robertocesar@ufc.br}

\section{Funding sources \\ CNPq (Conselho Nacional de Desenvolvi- mento Científico e Tecnológico); CAPES (Coordenação de Aperfeiçoamento de Pessoal de Nivel Superior) and FUNCAP (Fundação Cearense de Apoio ao Desenvolvi- mento Científico e Tecnológico)}

Conflicts of interest

None to declare.

Accepted for publication

8 May 2012

doi:10.1002/j.1532-2149.2012.00177.x

\begin{abstract}
Background: Some studies have shown a somatic nociceptive response due to the activation of transient receptor potential Al channels (TRPAl), which is modulated by the TRPAl antagonist HC-030031. However, a few studies report the role of TRPAl in visceral pain. Therefore, we investigated the participation of TRPAl in visceral nociception and the involvement of nitric oxide, the opioid system and resident cells in the modulation of these channels.
\end{abstract}

Methods: Mice were treated with vehicle or HC-030031 (18.75-300 mg/ $\mathrm{kg}$ ) before ifosfamide $(400 \mathrm{mg} / \mathrm{kg}), 0.75 \%$ mustard oil $(50 \mu \mathrm{L} / \mathrm{colon})$, acetic acid $0.6 \%$ (10 mL/kg), zymosan ( $1 \mathrm{mg} /$ cavity) or misoprostol ( $1 \mu \mathrm{g} /$ cavity) injection. Visceral nociception was assessed through the electronic von Frey test or the writhing response. Ifosfamide-administered mice were euthanized for bladder analysis. The involvement of nitric oxide and the opioid system were investigated in mice injected with ifosfamide and mustard oil, respectively. The participation of resident peritoneal cells in acetic acid-, zymosan- or misoprostol-induced nociception was also evaluated.

Results: HC-030031 failed to protect animals against ifosfamide-induced bladder injury $(p>0.05)$. However, a marked antinociceptive effect against ifosfamide, mustard oil, acetic acid, zymosan and misoprostol was observed $(p<0.05)$. Neither L-arginine $(600 \mathrm{mg} / \mathrm{kg})$ nor naloxone $(2 \mathrm{mg} / \mathrm{kg})$ could reverse the antinociceptive effect of HC-030031. The reduction of the peritoneal cell population inhibited the acetic acid and zymosan-related writhes without interfering with the misoprostol effect.

Conclusions: Our findings suggest that the blockade of TRPAl attenuates visceral nociception by a mechanism independent of the modulation of resident cells, nitric oxide and opioid pathways.

\section{Introduction}

Visceral pain is one of the most frequent reasons why patients seek medical attention (Cervero and Laird, 1999) and the most common form of pain produced by diseases such as, gastric distention (Kondo et al., 2010), inflammatory bowel disease (Bueno et al.,
2007) and haemorrhagic cystitis (Lima-Júnior et al., 2007).

Considering the diverse nature of diseases, it is extremely difficult to design a model of visceral pain in laboratory animals that can exactly mimic all the characteristics of functional visceral pain (Sengupta, 2009). Experimental pain models for assessment of analgesic 


\section{What's already known about this topic?}

- TRPAl is involved in visceral pain.

- However, the precise mechanism by which TRPAl contributes to visceral pain is still unknown.

\section{What does this study add?}

- This study provides a pharmacological approach regarding the participation of TRPAl in visceral nociception and the possible mechanisms involved.

effect needs to be reproducible, valid and responding in a uniform way to changes in pain level (Staahl et al., 2006). Some pain models provide meaningful results that are directly applicable to the interpretation of clinical data (Giamberardino, 1999).

The role of transient receptor potential (TRP) channels in visceral pain has been broadly investigated (Cattaruzza et al., 2010). TRP channels are excitatory ion channels mainly expressed in nociceptive neurons and contribute to the detection of noxious mechanical, thermal and pro-inflammatory stimuli (McNamara et al., 2007; Eid et al., 2008; Wang et al., 2008; Kerstein et al., 2009). Currently, they have been considered as potential targets in the management of visceral and inflammatory pain. Members of the TRP vanilloid family, such as Transiente Receptor Potential Vanilloid 1 (TRPV 1), are widely involved in visceral hyperalgesia (Hobson and Aziz, 2007; Sipe et al., 2008). However, there are only a few reports regarding the participation of TRPAl on visceral nociception (Yang et al., 2008; Cattaruzza et al., 2010; Kondo et al., 2010).

Transient receptor potential ankyrin-1 (TRPAl) is expressed in sensory nerves and mediates cold, mechanical and chemical nociception (Story et al., 2003; Bandell et al., 2004; Jordt et al., 2004). It is directly activated by exogenous alimentary (e.g., mustard oil) and environmental (e.g., acrolein) irritants (Bautista et al., 2006).

Notably, TRPAl is expressed in visceral afferent neurons and is known to participate in inflammatory responses and the establishment of hypersensitivity (Cattaruzza et al., 2010). It has been reported that inflammatory agents can directly activate or indirectly sensitize TRPAl to induce somatic pain and hyperalgesia (Petrus et al., 2007; Andrade et al., 2008; Wang et al., 2008; Liang et al., 2010; Fernandes et al., 2011). Wang et al. (2008) revealed that bradykinin might trigger the sensation of pain through TRPAl activation by a phospholipase C-dependent mechanism-
.However, the precise mechanism by which TRPAl contributes to visceral pain and inflammation is still unknown. Some hypothesis have been tested, including the role of TRPAl on substance $\mathrm{P}$ release from the primary sensory neurons through phosphorylation of p38 (Nakamura et al., 2012), and the release of histamine by mast cells (Andrade et al., 2008). Furthermore, non-neuronal cells on which TRPVI and TRPAl are expressed vary from vascular smooth muscle to keratinocytes and endothelium (Fernandes et al., 2012), which suggests a broader role of TRPAl.

Therefore, the present research investigated the role of TRPAl in visceral nociception and the participation of nitric oxide (NO), the opioid system and resident cells in the modulation of these channels.

\section{Methods}

\subsection{Animals}

Male Swiss mice weighing $20-25 \mathrm{~g}$ were kept in a temperature-controlled room $\left(22 \pm 2{ }^{\circ} \mathrm{C}\right)$ under 12 -h dark-light cycles with free access to food and water. The animals were fasted $18 \mathrm{~h}$ before the experiments. The Local Ethics Committee for Animal Experiments approved the entire protocol (number 92/10), complying to IASP's guidelines for pain research in animals (Zimmermann, 1983). All the experiments performed in this research were conducted by a blinded person who was unaware of the treatments and group divisions.

\subsection{Drugs}

HC-030031 was kindly provided by Hydra Biosciences, Inc. (Cambridge, MA, USA). For all experiments, HC-030031 was suspended in 0.5\% carboximethylcellulose $(0.5 \%$ CMC). Diazepam (Sanofi, São Paulo, SP, Brazil), indomethacin (Sigma-Aldrich, São Paulo, SP, Brazil), morphine (Cristália, São Paulo, SP, Brazil), naloxone (Cristália, São Paulo, SP, Brazil), mesna (Eurofarma, São Paulo, SP, Brazil), Ifosfamide (Eurofarma, São Paulo, SP, Brazil), misoprostol (Herbron, Caruaru, PE, Brazil), L-NAME (Sigma-Aldrich, São Paulo, SP, Brazil), L-Arginine (Sigma-Aldrich, São Paulo, SP, Brazil), mustard oil (Sigma-Aldrich, São Paulo, SP, Brazil), acetic acid and zymosan (SigmaAldrich, São Paulo, SP, Brazil) were dissolved in sterile saline $(0.9 \%)$.

\subsection{Induction and cystitis quantification}

To study the anti-inflammatory and the antinociceptive effects of HC-030031 on ifosfamide-induced 
haemorrhagic cystitis, groups of mice $(n=8)$ were treated with HC-030031 (75, 150 or $300 \mathrm{mg} / \mathrm{kg}$, p.o.) or vehicle $(0.5 \%$ CMC in distilled water) $60 \mathrm{~min}$ before the intraperitoneal injection of ifosfamide (IFO, $400 \mathrm{mg} / \mathrm{kg}$ ). The animals were euthanized $12 \mathrm{~h}$ later. The bladder was removed by careful dissection, emptied of urine, weighed to quantify vesical oedema (increase in bladder wet weight expressed as mg/20 g body weight) and evaluated for the macroscopic and microscopic parameters described previously (Gray et al., 1986). Briefly, bladders were macroscopically examined for oedema and haemorrhage in a blinded manner. According to Gray's scoring criteria (Gray et al., 1986), oedema was considered severe (3+) when fluid was seen externally and internally in the bladder walls, moderate $(2+)$ when confined to the internal mucosa, mild $(1+)$ between normal and moderate and normal (0) when no oedema was observed. Haemorrhage was scored as follows: 3+, intravesical clots; 2+, mucosal haematomas; $1+$, telangiectasia or dilatation of bladder vessels; and 0 , normal.

Bladders were fixed in formalin for $12 \mathrm{~h}$, dehydrated in alcohol and embedded in paraffin. Serial $5-\mu \mathrm{m}$ sections were stained with haematoxylin and eosin for analysis of the lesions. Histopathological analysis was performed by a blinded person who was unaware of the treatments and group divisions, and scored as follows: (0) normal epithelium and absence of inflammatory cell infiltration and ulceration; (1) mild changes involving reduction of urothelial cells, flattening with submucosal oedema, mild haemorrhage and few ulcerations; (2) moderate changes including mucosal erosion, inflammatory cell infiltration, fibrin deposition, haemorrhage and multiple ulcerations; and (3) severe changes of previously described parameters.

\subsection{Evaluation of sensitivity to mechanical stimulation of the abdomen}

Electronic von Frey test was performed to assess visceral nociception after injection of ifosfamide. The apparatus consists of acrylic boxes $(12 \times 20 \times 17 \mathrm{~cm})$ with a floor grid, which is viewed through a mirror positioned below, allowing observation of animals. A digital analgesy meter connected to a force transducer with a $0.7 \mathrm{~mm}^{2}$ polypropylene tip (electronic von Frey) detects the intensity of stimulation (grams) applied to animals. We considered the appearance of any of the following behaviours on application of the tip as a withdrawal response: (1) sharp retraction of abdomen; (2) immediate licking or scratching of site of application of the tip; (3) jumping; or (4) flinching the hind paw (Laird et al., 2001). Care was taken not to stimulate the same point in succession to avoid 'windup' effects or desensitization. We also avoided stimulation in the area of the external genitalia and concentrated on the lower to mid abdomen (Laird et al., 2001). We obtained the readings of nociceptive responses before animal treatment with HC-030031 (baseline or $\mathrm{T} 0$ ) and $12 \mathrm{~h}$ (T1) after the intraperitoneal administration of saline or ifosfamide. The results were obtained in grams for the difference (T0-T1) between the applied forces [mean \pm standard error of mean (SEM)].

\subsection{Mustard oil-induced colonic nociception}

To assess the antinociceptive effect HC-030031 on mustard oil-induced colonic nociception, groups of mice groups $(n=8)$ were treated with HC-030031 (18.75, 37.0 or $75 \mathrm{mg} / \mathrm{kg}$, p.o.) or vehicle $(0.5 \%$ CMC in distilled water) $60 \mathrm{~min}$ before the intracolonic administration of mustard oil (Sigma-Aldrich, São Paulo, SP, Brazil; $0.75 \%$ in $0.9 \%$ saline, $50 \mu \mathrm{L} /$ animal). A group of controls received a similar volume of saline $(50 \mu \mathrm{L} / \mathrm{colon})$. The sensitivity to mechanical stimulation of the abdomen was measured before animal treatment with HC-030031 (baseline or T0) 10 (T1) min after mustard oil instillation.

\subsection{Writhing test}

The writhing test was performed as previously described (Collier et al., 1968). Briefly, mice treated with $0.9 \%$ sterile saline ( $10 \mathrm{~mL} / \mathrm{kg}$, i.p.) or HC-030031 (18.75; 37.5 or $75.0 \mathrm{mg} / \mathrm{kg}$, p.o.) were administered acetic acid $0.6 \%$ (10 mL/kg, i.p.), zymosan ( $1 \mathrm{mg} / \mathrm{mL} /$ cavity, i.p.) or misoprostol ( $1 \mu \mathrm{g} / 0.2 \mathrm{~mL} /$ cavity, i.p.) into the peritoneal cavity. The intensity of nociception was quantified by counting the cumulative number of writhes occurring between 0 and 30 min after stimulus injection.

\subsection{Depletion of total peritoneal resident cells by peritoneal lavage}

Swiss mice were anaesthetized with tribromoethanol and three hypodermic needles were inserted into the abdominal cavity. Sterile saline $10 \mathrm{~mL}$ was injected through the needle placed near the sternum. The abdominal cavity was then gently massaged for $1 \mathrm{~min}$, and the peritoneal fluid was collected via the two needles inserted into the inguinal region. This operation was repeated three times. Control (sham) mice underwent insertion of the three needles and were 
Table 1 HC-030031 does not protect against ifosfamide-induced haemorrhagic cystitis.

\begin{tabular}{|c|c|c|c|c|}
\hline \multirow[b]{2}{*}{ Experimental groups } & \multirow[b]{2}{*}{ Bladder wet weight } & \multicolumn{2}{|c|}{ Macroscopic analysis } & \multirow[b]{2}{*}{ Microscopic analysis } \\
\hline & & Oedema & Haemorrhage & \\
\hline Saline (10 mL/kg) & $20.3 \pm 0,8$ & $0(0-0)$ & $0(0-0)$ & $0(0-0)$ \\
\hline Ifosfamide (IFO 400 mg/kg) & $44.0 \pm 3,2^{\star}$ & $2(1-3)^{*}$ & $3(1-3)^{\star}$ & $2,5(1-3)^{*}$ \\
\hline HC-030031 (75 mg/kg) + IFO & $55.6 \pm 5,1$ & $2(1-3)$ & $2,5(0-3)$ & $3(2-3)$ \\
\hline HC-030031 (150 mg/kg) + IFO & $39.1 \pm 4,7$ & $1(0-3)$ & $1,5(0-3)$ & $3(2-3)$ \\
\hline HC-030031 (300 mg/kg) + IFO & $50.5 \pm 7,7$ & $1,5(1-3)$ & $2(0-3)$ & $3(3-3)$ \\
\hline Mesna $(80 \mathrm{mg} / \mathrm{kg})+\mathrm{IFO}$ & $27.0 \pm 2,6 * \star$ & $0(0-2) * \star$ & $0(0-2)^{\star *}$ & $1(0-2)^{\star \star}$ \\
\hline
\end{tabular}

Data are presented as mean \pm SEM for bladder wet weight or median values (minimum and maximum) for macroscopic and microscopic scores. Analyses were performed $12 \mathrm{~h}$ after ifosfamide injection (ANOVA and Student Newman-Keul's test - parametric data; Kruskal-Wallis and Dunn's test - nonparametric data).

${ }^{*} p<0.05$ versus saline.

$\star * p<0.05$ versus IFO

manipulated similarly, but no fluid was injected or withdrawn. Thirty minutes after recovering from sedation, the animals were injected with acetic acid $0.6 \%(10 \mathrm{~mL} / \mathrm{kg})$, zymosan ( $1 \mathrm{mg} /$ cavity) or misoprostol $(1 \mu \mathrm{g} /$ cavity), and the number of writhes was counted in the interval between 0 and 30 min after injection of the stimuli (Souza et al., 1988).

\subsection{Prostaglandin $\mathrm{E}_{\mathbf{2}}\left(\mathrm{PGE}_{2}\right)$-induced licking paw}

$\mathrm{PGE}_{2}$ ( $3 \mathrm{nmol} / 20 \mu \mathrm{L}$ per paw) or saline (vehicle per paw) was injected under the plantar surface of the right hind paw (i.pl. injection) $60 \mathrm{~min}$ after HC-030031 (75 mg/kg, p.o.). The animals were allowed to individually adapt to the experimental area (transparent glass cylinders, $20 \mathrm{~cm}$ in diameter) for at least 20 min before algogen or vehicle injection. After injection, the mice were observed individually for $15 \mathrm{~min}$, and the total time spent licking the injected paw recorded (Kassuya et al., 2007).

\subsection{Open-field test}

Mice were observed for spontaneous locomotion in an open-field apparatus for a period of $4 \mathrm{~min}$. This test was performed $60 \mathrm{~min}$ after of oral administration of HC-030031 $(75 \mathrm{mg} / \mathrm{kg})$, vehicle $(0.5 \%$ CMC in distilled water) or diazepam ( $5 \mathrm{mg} / \mathrm{kg}$; Capaz et al., 1981).

\subsection{Statistical analysis}

Data were subjected to Student's t-test for unpaired values, One-way analysis of variance followed by Student Newman-Keul's test or Kruskal-Wallis followed by Dunn's test, as appropriate. Values were expressed as mean \pm SEM (parametric data) or median (minimum-maximum) for non-parametric data. The level of significance was set at $p<0.05$.

\section{Results}

\subsection{HC-030031 is devoid of anti-inflammatory effect}

Table 1 shows that ifosfamide induced a significant increase in bladder wet weight, macroscopic (oedema and haemorrhage) and microscopic scores when compared with saline $(p<0.05)$. HC-030031, in all doses tested $(75,150$ and $300 \mathrm{mg} / \mathrm{kg}, \mathrm{p} . \mathrm{o})$, failed to protect the animals against ifosfamide-induced damage $(p>0.05)$. In addition, the standard control, mesna ( $80 \mathrm{mg} / \mathrm{kg}$, i.p.), significantly protected mice from the increase in bladder wet weight and the macroscopic (oedema and haemorrhage) and microscopic scores compared to the ifosfamide-injected group $(p<0.05)$.

\subsection{Visceral antinociceptive effect of HC-030031}

Fig. 1A shows that ifosfamide induced a significant increase in visceral nociception when compared with saline $(p<0.05)$. Additionally, HC-030031, in all doses tested $(75,150$ and $300 \mathrm{mg} / \mathrm{kg}, \mathrm{p.o})$, and the standard control, mesna (80 mg/kg, i.p.), significantly protected mice from visceral nociception development $(p<0.05)$. Similar nociceptive response was found when mustard oil was administered into the colon of mice compared to saline $(p<0.05$, Fig. 1C). Such response was significantly inhibited when mice were administered HC-030031 (37.5 and $75 \mathrm{mg} / \mathrm{kg}$ ). We also observed that pretreatment with L-NAME (10 mg/kg, Fig. 1B) and morphine ( $5 \mathrm{mg} / \mathrm{kg}$, Fig. 1D) prevented ifosfamide and mustard oil-induced nocice- 
A

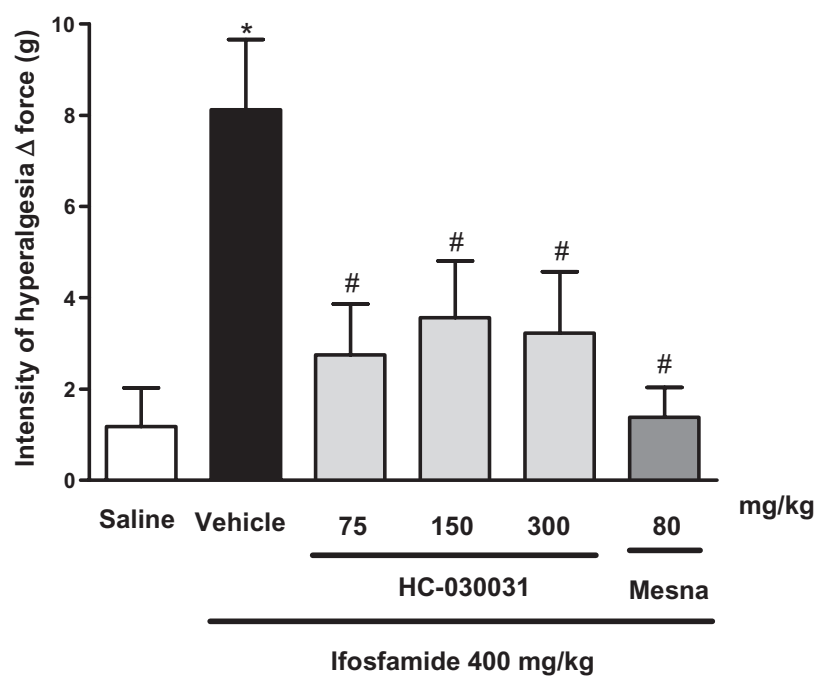

$\mathrm{C}$

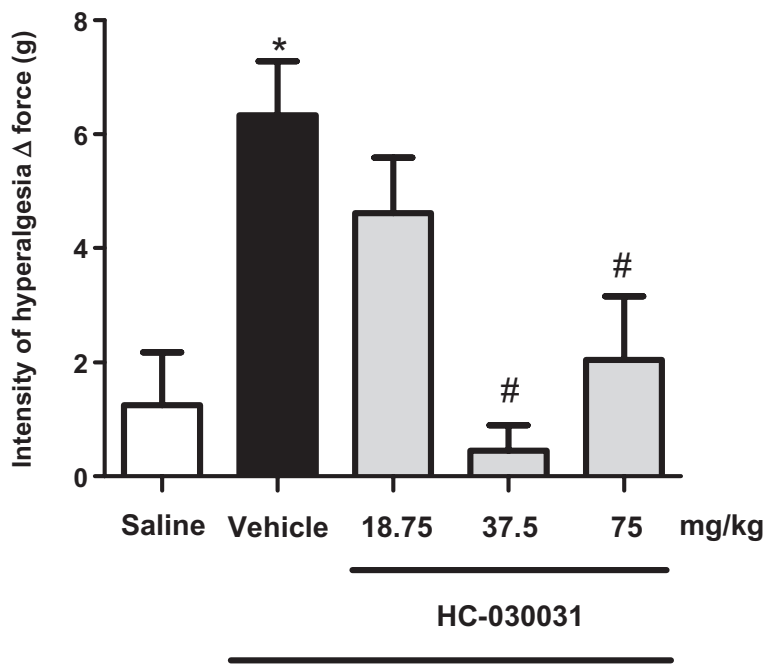

$0.75 \%$ Mustard oil
$B$

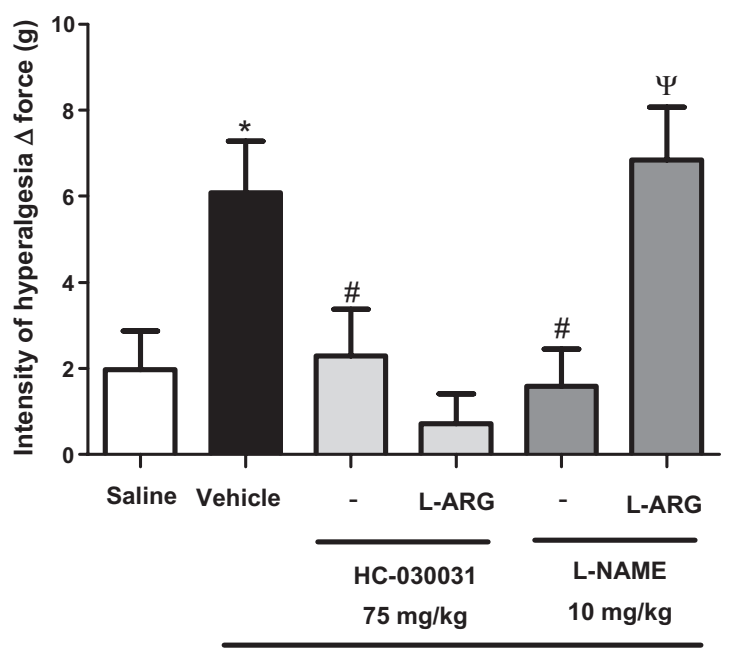

Ifosfamide $400 \mathrm{mg} / \mathrm{kg}$

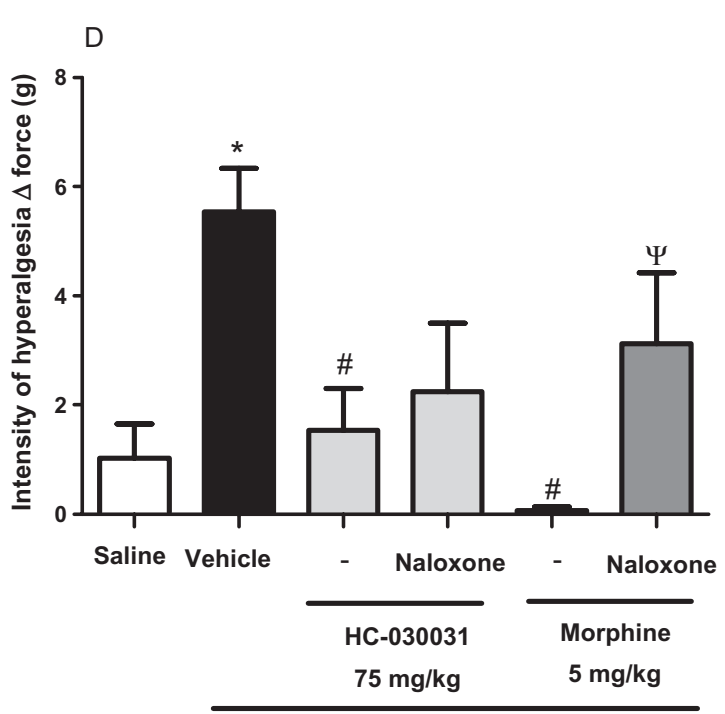

$0.75 \%$ Mustard oil

Figure 1 Effect of HC-030031 on visceral nociception: study of nitric oxide and opioid system involvement. The nociceptive response was assessed by the electronic von Frey test before animal treatments and $12 \mathrm{~h}$ after ifosfamide administration (A, B) or 10 min following mustard oil injection (C, D). Visceral nociception was prevented by HC-030031, which was unaffected by L-arginine (B) or naloxone pretreatment (D). The bars represent mean \pm SEM of 6-8 mice. Intensity of hyperalgesia was expressed in grams (g). ${ }^{*} p<0.01$ compared to saline group, ${ }^{*} p<0.05$ versus ifosfamide or mustard oil group, $\Psi p<0.05$ in comparison with L-NAME or morphine (ANOVA and Student Newman-Keul's test).

ption, respectively, which was markedly reverted $(p<0.05)$ by L-arginine $(600 \mathrm{mg} / \mathrm{kg})$ and naloxone $(2 \mathrm{mg} / \mathrm{kg})$. However, neither L-arginine nor naloxone affected the antinociceptive effect of HC-030031 $(p>0.05)$.

We further investigated the protective effect of HC-030031 in three viscero-somatic models of nociception using the writhing test initiated by different algogens. We found (Fig. 2A-C) that acetic acid, zymosan and misoprostol evoked pain-related behaviours $(42.43 \pm 3.07 ; 8.66 \pm 2.17$; and $5.57 \pm 1.19$, respectively), which were significantly inhibited by HC-030031 $(p<0.05)$ in the doses tested $(18.75,37.5$ and $75 \mathrm{mg} / \mathrm{kg}$ ). The maximal inhibitory potential varied according to the algogenic substance used and was higher against zymosan $(84.1 \%$, Fig. 2B) and 


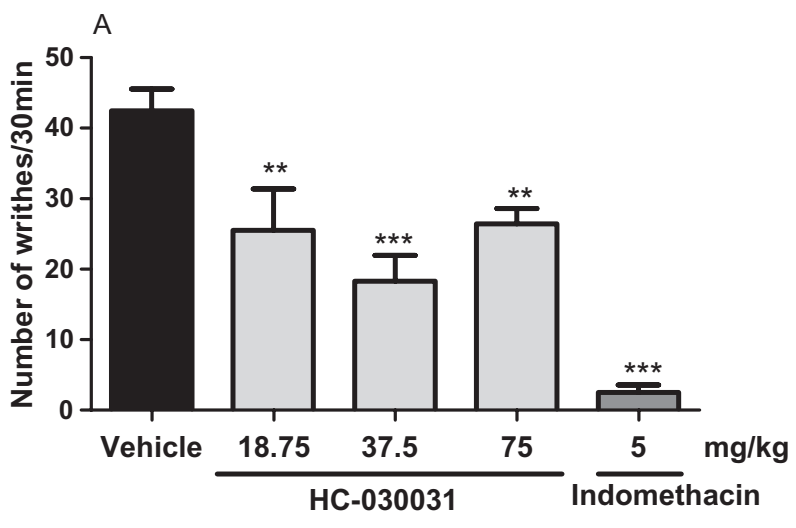

Acetic acid $0.6 \%$

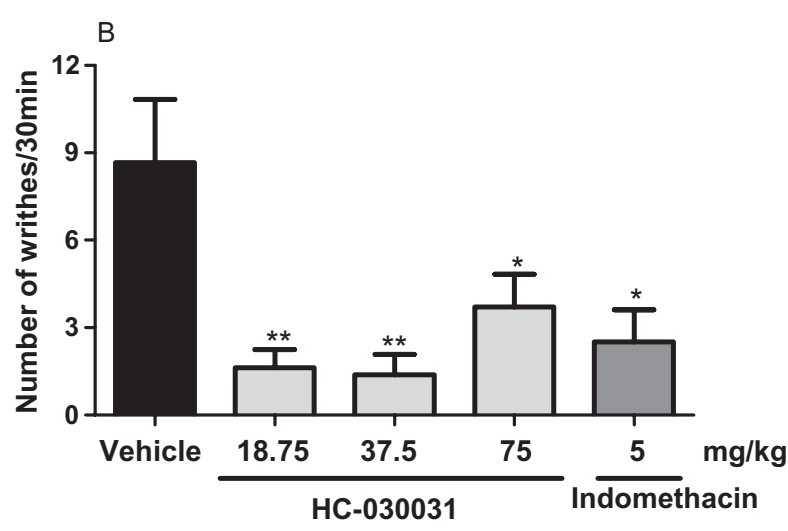

Zymosan (1 mg/cavity)

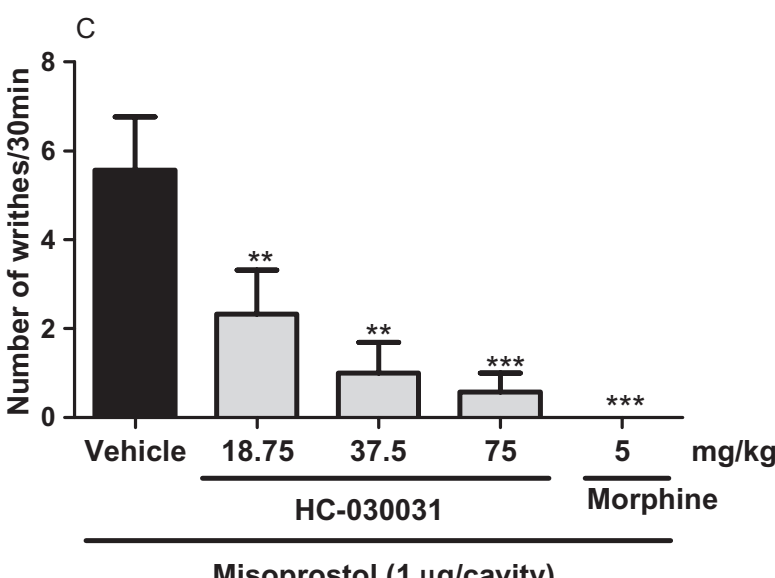

Figure 2 Antinociceptive effect of HC-030031 on acetic acid, zymosan and misoprostol-induced writhing behaviour. Swiss mice were pretreated with CMC, HC-030031, indomethacin or morphine 60 min previously the i.p. instillation of acetic acid (A), zymosan (B) or misoprostol (C). The bars represent mean \pm SEM of the writhing behaviours expressed during 30 min $(n=8) .{ }^{*} p<0.05,{ }^{* *} p<0.01$ and ${ }^{* * *} p<0.001$ versus vehicle group (ANOVA and Student Newman-Keul's test).

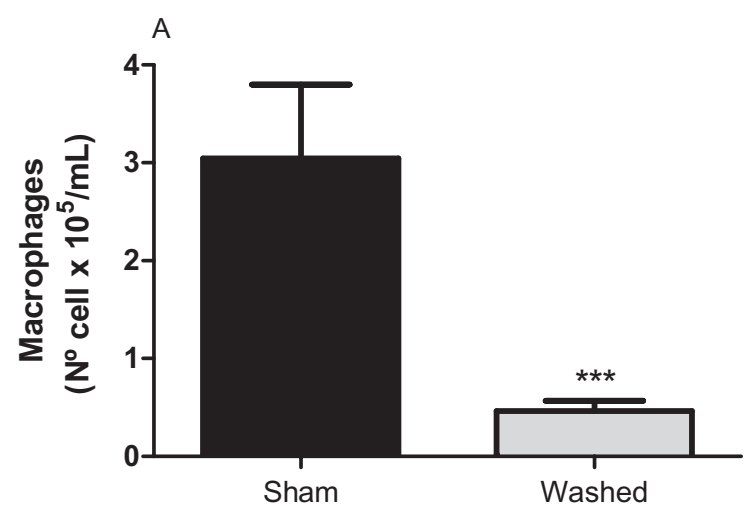

B

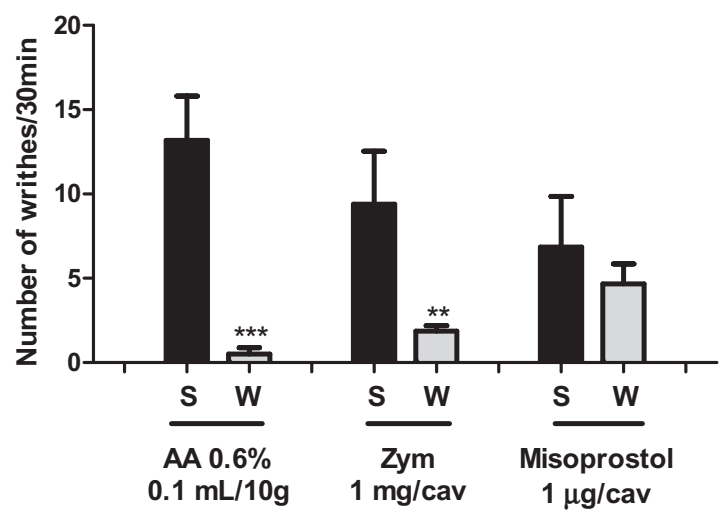

Figure 3 Effect of resident peritoneal cell depletion on acetic acid, zymosan or misoprostol-induced writhing behaviour. Anaesthetized mice had the peritoneal cavity perfused (W) with sterile saline. A no-washed group was included (sham). Following this procedure, heparinized PBS was instilled i.p. to perform the peritoneal cell count (A). Sham (S) and washed (W) groups $(n=8)$ were injected with acetic acid, zymosan or misoprostol. The number of writhes was recorded for $30 \mathrm{~min}$ (B). The bars represent mean \pm SEM. ${ }^{* *} p<0.01$ and ${ }^{* * *} p<0.001$ compared to sham group (unpaired Student's $t$-test).

misoprostol (89.7\%, Fig. 2C) than acetic acid (57\%, Fig. 2A). As positive controls for inhibition of nociception, indomethacin (in acetic acid- and zymosaninduced writhes) and morphine (misoprostol-induced nociception) showed markedly $(p<0.05)$ protective effects of $94 \%, 71.1 \%$ and $100 \%$, respectively (Fig. 2A-C).

The role of resident macrophages in the writhing response was evaluated by washing the peritoneal cavity. To test the efficacy of this procedure, we first counted the number of cells in the peritoneum lavage. Fig. 3A shows a pronounced reduction $(p<0.0004)$ in peritoneal macrophage population $(84.87 \%)$ when compared to non-washed cavities. Fig. 3 also shows that peritoneal-washed mice are less responsive $(p<0.05)$ to acetic acid $(0.50 \pm 0.38)$ and zymosan $(1.86 \pm 0.34)$ stimuli compared to sham animals 


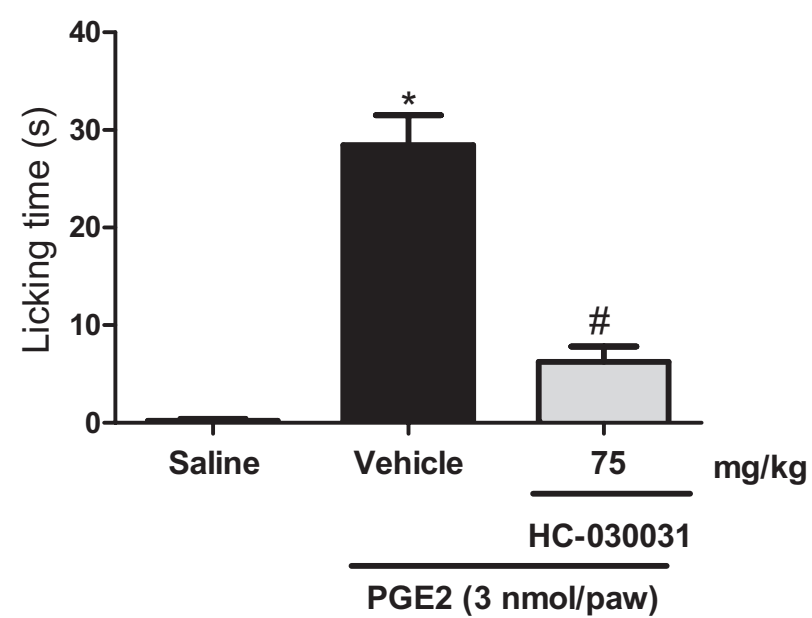

Figure 4 Effect of $\mathrm{HC}-030031$ on paw $\mathrm{PGE}_{2}$-induced nociception. Vehicle or $\mathrm{HC}-030031$ treated animals were injected with saline or $\mathrm{PGE}_{2}$ into the hind paw. Bars represent mean \pm SEM time in seconds mice spent licking the injected paws. ${ }^{*} p<0.001$ versus saline group and ${ }^{*} p<0.01$ versus $\mathrm{PGE}_{2}$ group (ANOVA and Student Newman-Keul's test).

(acetic acid: $13.17 \pm 2.64 ;$ zymosan: $9.40 \pm 3.14$, Fig. 3B). However, in spite of the peritoneal lavage procedure, misoprostol was able to elicit similar nociceptive responses compared to the sham group $(p>0.05$, Fig. 3B).

We further investigated whether HC-030031 would have an effect against a known direct nociceptor activator. As observed in Fig. 4, intraplantar $\mathrm{PGE}_{2}$ caused a significant nociceptive behaviour compared to saline-injected mice $(p<0.001)$. Pretreatment with HC-030031 (75 mg/kg) significantly decreased the total time mice spent licking the $\mathrm{PGE}_{2}$-injected paw (a $78.02 \%$ inhibition, $p<0.001)$.

\subsection{Effect on locomotor activity in open-field test}

To rule out the possible locomotor impairment due to HC-030031 treatment, we performed the open-field test. HC-030031 administration did not affect locomotion (Fig. 5) compared to vehicle-treated mice $(p>0.05)$. However, diazepam, a known sedative drug used as a positive control, significantly reduced $(p<0.01)$ the number of fields explored by the animals (41.74\% inhibition, Fig. 5).

\section{Discussion and conclusions}

In this study, HC-030031, a TRPAl receptor antagonist, was shown to have antinociceptive effects on different models of visceral pain. In addition, no antiinflammatory activity was observed for that substance.

Haemorrhagic cystitis is a common inflammatory condition due to bladder accumulation of acrolein, an urotoxic substance produced by liver metabolism of the anticancer agents, cyclophosphamide and ifosfamide (Cox, 1979). Some studies report that acrolein is a potential activator of TRPAl receptors (Bautista et al., 2006). Thus, we initially investigated whether the ifosfamide-induced inflammatory response would be dependent on TRPAl receptors. We observed that $\mathrm{HC}-030031$, even at doses as high as $300 \mathrm{mg} / \mathrm{kg}$, did not inhibit bladder damage. However, mesna, the only clinically available drug used to prevent haemorrhagic cystitis, afforded a clear anti-inflammatory effect. Therefore, haemorrhagic cystitis-associated inflammation is unlikely related to TRPAl activation by acrolein, but it is probably related to direct tissue damage and resident cell stimulation by this metabolite. However, the role of TRPAl in inflammation is still possible since it is expressed in capsaicin-sensitive primary afferent neurons known to contribute to neurogenic inflammation that result in increased vascular permeability and oedema formation (Silva et al., 2011).

Patients with bladder injury due to cancer treatment may also exhibit abdominal pain. Then, we further tested the antinociceptive activity of HC-030031 in the model of haemorrhagic cystitis-induced nociception (Olivar and Laird, 1999). We found that HC-030031 markedly inhibited ifosfamide-induced nociception, possibly suggesting that acrolein would be responsible for TRPAl activation (Bautista et al., 2006).

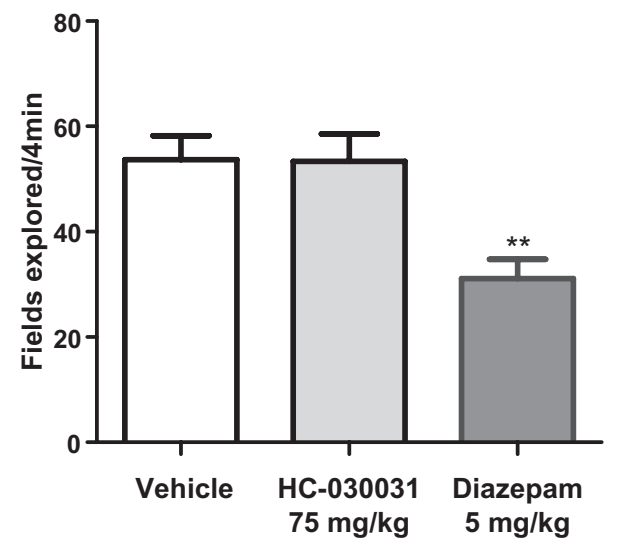

Figure 5 HC-030031 does not cause ambulation impairment. Vehicle, HC-030031 or diazepam treated mice $(n=8)$ were observed for 4 min regarding the ambulation in the open-field arena. ${ }^{* *} p<0.01$ compared to vehicle group. The bars represent the mean \pm SEM number of fields explored (ANOVA and Student Newman-Keul's test). 
Considering the well-known participation of NO in the haemorrhagic cystitis animal model (Souza-Filho et al., 1997), we decided to use such animal model to study the involvement of this mediator in the antinociceptive effect of HC-030031. It has been suggested that endogenous NO is involved in the inflammatory events leading to haemorrhagic cystitis after cyclophosphamide administration. Additionally, the role for peripherally acting NO in hyperalgesia is supported by pharmacological data (reviewed by Miyamoto et al., 2009). Furthermore, Miyamoto et al. (2009) provided genetic and pharmacological evidence that NO donors activate TRPVl and TRPAl both in heterologous systems and in naïve dorsal root ganglion (DRG) neurons. Thus, we investigated whether L-arginine, a NO synthesis precursor, would be able to reverse the effect of HC-030031 upon noxious stimulation due to ifosfamide injection. We found that a high dose of L-arginine failed in that regard. However, L-NAME administration led to a prominent antinociceptive activity, which was prevented by L-arginine pretreatment. The present finding is the first report regarding the role of NO in ifosfamide-induced visceral nociception. Notwithstanding previous results, we cannot rule out the modulation of TRPAl function by inflammatory mediators. A recent published article suggests that endogenous activation of TRPAl plays a critical role in the development of tumour necrosis factoralpha (TNF- $\alpha)$-induced mechanical hyperalgesia (Fernandes et al., 2011). Wang et al. (2008) also suggested that bradykinin released in response to tissue inflammation might trigger the sensation of pain by TRPAl activation. It is also proposed that TRPAl is involved in ET-1-induced spontaneous pain-like behaviour in mice (Liang et al., 2010). Therefore, the pathways by which TRPAl is activated in haemorrhagic cystitis merit further investigation.

We further evaluated the protective effect of HC-030031 in the model of mustard oil-induced visceral nociception (Laird et al., 2001). Mustard oil is a known TRPAl activator (Macpherson et al., 2007). Cattaruzza et al. (2010) reported that TRPAl is expressed by DRG neurons that innervate the colon and that TRPAl mediates inflammatory hyperalgesia of the colon. In our study, we showed that even low doses of the TRPAl blocker, HC-030031 (37.5 and $75 \mathrm{mg} / \mathrm{kg}$ ), significantly inhibited the noxious response elicited by the intracolonic instillation of mustard oil. These low doses, which are in a 1:2 dilution scale, were chosen considering the pronounced result found in the haemorrhagic cystitis model of nociception. Our results are corroborated by previous results, which demonstrated that higher doses of
HC-03003l (300 mg/kg) reduce the spinal c-Fos response to intracolonic allyl isothiocyanate (AITC), the active compound of mustard oil (Mitrovic et al., 2010). This indicates that the effect of AITC is mediated by TRPAl. Kondo et al. (2010) suggested that in response to noxious gastric distension, generator potentials mediated via TRPAl may promote peripheral action potentials, which in turn result in the phosphorylation of extracellular signal-regulated kinases 1 and 2 (ERK 1/2) in DRG and/or spinal neurons leading to visceral pain. In a review published by Engel et al. (2011), it is reported that the continuous sensitization of TRPAl by inflammatory mediators is found to be essential for colitis induction in the model of TNBS colitis through the release of neuropeptides. These neuronal mediators could be responsible for maintaining the inflammatory process (Engel et al., 2011) and hyperalgesia (Kondo et al., 2010).

It is currently unknown whether the antinociceptive action of HC-030031 would partially involve the opioid system. Taking into account that morphine effectively inhibits the noxious response due to the intracolonic instillation of mustard oil (Laird et al., 2001), we used this animal model to evaluate the participation of the opioid system on the antinociceptive effect of HC-030031. To test this hypothesis, we treated animals with the opioid receptor antagonist, naloxone, before TRPAl blocker administration. As observed, no reversal effect was detected. However, the effect of the positive control, morphine, was prevented by the opioid receptor antagonist. These findings possibly exclude the participation of the opioid system in the antinociceptive action of HC-030031.

We also evaluated the antinociceptive effect of HC-030031 in a viscero-somatic model of nociception (Laird et al., 2001) induced by three different algogens: acetic acid, zymosan and misoprostol, a $\mathrm{PGE}_{1}$ stable analogue. We found that $\mathrm{HC}-030031$ in all doses tested markedly inhibited the noxious response elicited by these agents. The literature reports that the release of TNF- $\alpha$ initiates the release of interleukin- $1 \beta$ and interleukin-8, which in turn liberate cyclooxygenase metabolites and sympathomimetic amines, respectively (Ferreira, 1993). Both cyclooxygenase products and sympathomimetic amines play a role in the development of the persistent nociceptor hypersensitivity induced by TNF- $\alpha$ (Ferreira, 1993; Sachs et al., 2002).

In a paper published by Ribeiro et al. (2000), the authors clearly suggested that the nociceptive activity of zymosan and acetic acid in the writhing model may be due to the release of TNF- $\alpha$, interleukin-1 $\beta$ and interleukin- 8 by resident peritoneal macrophages and 
mast cells. Such conclusions were partially achieved by reducing or enhancing the peritoneal cell population. We similarly verified the mild nociceptive activities of zymosan and acetic acid in animals depleted of peritoneal resident cells. In addition, indomethacin, a non-specific cyclooxygenase inhibitor, markedly prevented the zymosan and acetic acid-related writhes, suggesting that neosynthesized prostaglandins are involved in this nociceptive effect. We also evaluated the contribution of peritoneal cells to misoprostolinduced nociception. Accordingly fitting with results reported by Ribeiro et al. (2000) using iloprost, a stable analogue of prostacyclin, we showed that lavage of the cavities did not affect the nociceptive response to misoprostol. Therefore, these results suggest that misoprostol, like iloprost, may activate the nociceptor directly (Ferreira et al., 1978). Besides, morphine, a potent nociceptor stabilizer, was able to prevent neuron activation due to misoprostol injection. To support the suggestion that HC-030031 may stabilize the nociceptor directly, we further investigated its effect in the $\mathrm{PGE}_{2}$-induced paw nociception (Kassuya et al., 2007). In accordance, HC-030031 significantly prevented $\mathrm{PGE}_{2}$-induced nociceptive licking paw behaviour.

Taking into account that the sensitization of primary sensory neurons involves an inflammatory cascade, the modulation of nociception could be reached by the inhibition of any intermediate mediator, including TNF- $\alpha$, or by stabilising the nociceptor directly. In our opinion, HC-030031 would be acting through the later mechanism due to two main reasons: (1) The nociceptive effect of $\mathrm{PGE}_{2}$ and of a $\mathrm{PGE}_{1}$ stable analogue, misoprostol, was both abolished by HC-030031. Therefore, the antinociceptive activity of HC-030031 is not due to an inhibitory effect on prostaglandin release; (2) peritoneal lavage did not prevent the nociceptive capacity of misoprostol. Thence, in this experimental condition, misoprostol did not induce the release of inflammatory mediators by resident peritoneal cells. Altogether, these data point out that the antinociceptive action of HC-030031 results from direct nociceptor stabilization and seems to be independent of the inhibition of inflammatory resident cells.

In addition, our study showed that HC-030031 did not impair locomotion performance in open-field tests with mice. However, diazepam, a sedative drug adopted as a positive control, markedly inhibited mice locomotion. Eid et al. (2008) also demonstrated that HC-030031, even at higher doses $(300 \mathrm{mg} / \mathrm{kg}$ ), failed to have any effect on rotarod response. These results indicate that the antinociceptive effect of this TRPAl antagonist is not due to animal sedation.
In summary, our results suggest that the antinociceptive effect of HC-030031 in various animal models of visceral pain seems to be independent of the inhibition of inflammatory resident cells, NO and opioid pathways. We also showed that HC-030031 likely acts as a direct nociceptor stabilizer. This study provides perspective for the effective management of visceral pain through the modulation of TRPAl channels. However, the mechanisms that underlie the involvement of TRPAl receptors and its regulation by inflammatory mediators need further investigation.

\section{Author contributions}

A.X.C.B., C.G.T., L.S.G., R.P.M., R.D.M.N., R.B.C., K.S.A. and D.V.T.W.: execution of the project; L.M.S.P., M.L.V.: execution of the project, data analysis and wrote the manuscript; G.A.C.B.: microscopic analysis; G.A.C.B., R.C.P.L.J. and R.A.R.: designed the experiments, data analysis, interpreted the results and wrote the manuscript. All authors discussed the results and commented on the manuscript.

\section{Acknowledgements}

We are grateful to Maria Silvandira Freire and Karina Felismino da Silva Santos for technical assistance.

\section{References}

Andrade, E.L., Luiz, A.P., Ferreira, J., Calixto, J.B. (2008). Pronociceptive response elicited by TRPAl receptor activation in mice. Neuroscience 152(2), 511-520.

Bandell, M., Story, G.M., Hwang, S.W., Viswanath, V., Eid, S.R., Petrus, M.J., Earley, T.J., Patapoutian, A. (2004). Noxious cold ion channel TRPAl is activated by pungent compounds and bradykinin. Neuron 41(6), 849-857.

Bautista, D.M., Jordt, S.E., Nikai, T., Tsuruda, P.R., Read, A.J., Poblete, J., Yamoah, E.N., Basbaum, A.I., Julius, D. (2006). TRPAl mediates the inflammatory actions of environmental irritants and proalgesic agents. Cell 124(6), 1269-1282.

Bueno, L., de Ponti, F., Fried, M., Kullak-Ublick, G.A., Kwiatek, M.A., Pohl, D., Quigley, E.M., Tack, J., Talley, N.J. (2007). Serotonergic and non-serotonergic targets in the pharmacotherapy of visceral hypersensitivity. Neurogastroenterol Motil 19(1 Suppl), 89-119.

Capaz, F.R., Vanconcellos, L.E., De Moraes, S., Neto, J.P. (1981). The open field: A simple method to show ethanol withdrawal symptoms. Arch Int Pharmacodyn Ther 251(2), 228-236.

Cattaruzza, F., Spreadbury, I., Miranda-Morales, M., Grady, E.F., Vanner, S., Bunnett, N.W. (2010). Transient receptor potential ankyrin-1 has a major role in mediating visceral pain in mice. Am J Physiol Gastrointest Liver Physiol 298(1), G81-G91.

Cervero, F., Laird, J.M.A. (1999). Visceral pain. Lancet 353(9170), 2145-2148.

Collier, H.O., Dinneen, L.C., Johnson, C.A., Schneider, C. (1968). The abdominal constriction response and its suppression by analgesic drugs in the mouse. Br J Pharmacol Chemother $32(2), 295-310$. 
Cox, P.J. (1979). Cyclophosphamide cystitis - Identification of acrolein as the causative agent. Biochem Pharmacol 28(13), 2045-2049.

Eid, S.R., Crown, E.D., Moore, E.L., Liang, H.A., Choong, K.C., Dima, S., Henze, D.A., Kane, S.A., Urban, M.O. (2008). HC-030031, a TRPAl selective antagonist, attenuates inflammatory- and neuropathy-induced mechanical hypersensitivity. Mol Pain 4(48), 1-10.

Engel, M.A., Becker, C., Reeh, P.W., Neurath, M.F. (2011). Role of sensory neurons in colitis: Increasing evidence for a neuroimmune link in the gut. Inflamm Bowel Dis 17(4), 10301033.

Fernandes, E.S., Fernandes, M.A., Keeble, J.E. (2012). The functions of TRPAl and TRPV1: Moving away from sensory nerves. Br J Pharmacol 166(2), 510-521.

Fernandes, E.S., Russell, F.A., Spina, D., McDougall, J.J., Graepel, R., Gentry, C., Staniland, A.A., Mountford, D.M., Keeble, J.E., Malcangio, M., Bevan, S., Brain, S.D. (2011). A distinct role for transient receptor potential ankyrin 1, in addition to transient receptor potential vanilloid 1 , in tumor necrosis factor $\alpha$-induced inflammatory hyperalgesia and Freund's complete adjuvant-induced monarthritis. Arthritis Rheum 63(3), 819-829.

Ferreira, S.H. (1993). The role of interleukins and nitric oxide in the mediation of inflammatory pain and its control by peripheral analgesics. Drugs 46(Suppl 1), 1-9.

Ferreira, S.H., Lorenzetti, B.B., Correa, F.M.A. (1978). Central and peripheral antialgesic action of aspirin-like drugs. Eur $J$ Pharmacol 53(1), 39-48.

Giamberardino, M.A. (1999). Recent and forgotten aspects of visceral pain. Eur J Pain 3(2), 77-92.

Gray, K.J., Engelmann, U.H., Johnson, E.H., Fishman, I.J. (1986). Evaluation of misoprostol cytoprotection of the bladder with cyclophosphamide (Cytoxan) therapy. J Urol 136(2), 497-500.

Hobson, A.R., Aziz, Q. (2007). Modulation of visceral nociceptive pathways. Curr Opin Pharmacol 7(6), 593-597.

Jordt, S.E., Bautista, D.M., Chuang, H.H., McKemy, D.D., Zygmunt, P.M., HöGestäTt, E.D., Meng, I.D., Julius, D. (2004). Mustard oils and cannabinoids excite sensory nerve fibres through the TRP channel ANKTM1. Nature 427(6971), 260265.

Kassuya, C.A., Ferreira, J., Claudino, R.F., Calixto, J.B. (2007). Intraplantar PGE2 causes nociceptive behaviour and mechanical allodynia: The role of prostanoid E receptors and protein kinases. Br J Pharmacol 150(6), 727-737.

Kerstein, P.C., Del Camino, D., Moran, M.M., Stucky, C.L. (2009). Pharmacological blockade of TRPAl inhibits mechanical firing in nociceptors. Mol Pain 5(19), 1-13.

Kondo, T., Oshima, T., Obata, K., Sakurai, J., Knowles, C.H., Matsumoto, T., Noguchi, K., Miwa, H. (2010). Role of transient receptor potential Al in gastric nociception. Digestion $82(3), 150-155$.

Laird, J.M., Martinez-Caro, L., Garcia-Nicas, E., Cervero, F. (2001). A new model of visceral pain and referred hyperalgesia in the mouse. Pain 92(3), 335-342.

Liang, J., Bi, H., Ji, W. (2010). Involvement of TRPAl in ET-1induced pain-like behavior in mice. Neuroreport 21(3), 201205.

Lima-Júnior, R.C., Sousa, D.I., Brito, G.A., Cunha, G.M., Chaves, M.H., Rao, V.S., Santos, F.A. (2007). Modulation of acute visceral nociception and bladder inflammation by plant triterpene, alpha, beta-amyrin in a mouse model of cystitis:
Role of tachykinin NK(1)-receptors, and $\mathrm{K}(+)$ (ATP) channels. Inflamm Res 56(12), 487-494.

Macpherson, L.J., Dubin, A.E., Evans, M.J., Marr, F., Schultz, P.G., Cravatt, B.F., Patapoutian, A. (2007). Noxious compounds activate TRPAl ion channels through covalent modification of cysteines. Nature 445(7127), 541-545.

McNamara, C.R., Mandel-Brehm, J., Bautista, D.M., Siemens, J., Deranian, K.L., Zhao, M., Hayward, N.J., Chong, J.A., Julius, D., Moran, M.M., Fanger, C.M. (2007). TRPAl mediates formalin-induced pain. Proc Natl Acad Sci U S A 104(33), 13525-13530.

Mitrovic, M., Shahbazian, A., Bock, E., Pabst, M.A., Holzer, P. (2010). Chemo-nociceptive signalling from the colon is enhanced by mild colitis and blocked by inhibition of transient receptor potential ankyrin 1 channels. Br J Pharmacol 160(6), 1430-1442.

Miyamoto, T., Dubin, A.E., Petrus, M.J., Patapoutian, A. (2009). TRPVI and TRPAl mediate peripheral nitric oxide-induced nociception in mice. PLoS ONE 4(10), e7596.

Nakamura, Y., Une, Y., Miyano, K., Abe, H., Hisaoka, K., Morioka, N., Nakata, Y. (2012). Activation of transient receptor potential ankyrin 1 evokes nociception through substance P release from primary sensory neurons. J Neurochem 120(6), 1036-1047.

Olivar, T., Laird, J.M. (1999). Cyclophosphamide cystitis in mice: Behavioural characterisation and correlation with bladder inflammation. Eur J Pain 3(2), 141-149.

Petrus, M., Peier, A.M., Bandell, M., Hwang, S.W., Huynh, T., Olney, N., Jegla, T., Patapoutian, A. (2007). A role of TRPAl in mechanical hyperalgesia is revealed by pharmacological inhibition. Mol Pain 3(40), 1-8.

Ribeiro, R.A., Vale, M.L., Thomazzi, S.M., Paschoalato, A.B., Poole, S., Ferreira, S.H., Cunha, F.Q. (2000). Involvement of resident macrophages and mast cells in the writhing nociceptive response induced by zymosan and acetic acid in mice. Eur J Pharmacol 387(1), 111-118.

Sachs, D., Cunha, F.Q., Poole, S., Ferreira, S.H. (2002). Tumour necrosis factor-alpha, interleukin-lbeta and interleukin-8 induce persistent mechanical nociceptor hypersensitivity. Pain 96(1-2), 89-907.

Sengupta, J.N. (2009). Visceral pain: The neurophysiological mechanism. Handb Exp Pharmacol 194, 31-74.

Silva, C.R., Oliveira, S.M., Rossato, M.F., Dalmolin, G.D., Guerra, G.P., Da Silveira, P.A., Cabrini, D.A., Otuki, M.F., André, E., Ferreira, J. (2011). The involvement of TRPAl channel activation in the inflammatory response evoked by topical application of cinnamaldehyde to mice. Life Sci 88(25-26), 1077 1087.

Sipe, W.E., Brierley, S.M., Martin, C.M., Phillis, B.D., Cruz, F.B., Grady, E.F., Liedtke, W., Cohen, D.M., Vanner, S., Blackshaw, L.A., Bunnett, N.W. (2008). Transient receptor potential vanilloid 4 mediates protease activated receptor 2 -induced sensitization of colonic afferent nerves and visceral hyperalgesia. Am J Physiol Gastrointest Liver Physiol 294(5), G1288-G1298.

Souza, G.E., Cunha, F.Q., Mello, R., Ferreira, S.H. (1988). Neutrophil migration induced by inflammatory stimuli is reduced by macrophage depletion. Agents Actions 24(3-4), $377-380$

Souza-Fiho, M.V., Lima, M.V., Pompeu, M.M., Ballejo, G., Cunha, F.Q., Ribeiro, R. de A. (1997). Involvement of nitric oxide in the pathogenesis of cyclophosphamide-induced hemorrhagic cystitis. Am J Pathol 150(1), 247-256. 
Staahl, C., Reddy, H., Andersen, S.D., Arendt-Nielsen, L., Drewes, A.M. (2006). Multi-modal and tissue-differentiated experimental pain assessment: Reproducibility of a new concept for assessment of analgesics. Basic Clin Pharmacol Toxicol 98(2), 201-211.

Story, G.M., Peier, A.M., Reeve, A.J., Eid, S.R., Mosbacher, J., Hricik, T.R., Earley, T.J., Hergarden, A.C., Andersson, D.A., Hwang, S.W., McIntyre, P., Jegla, T., Bevan, S., Patapoutian, A. (2003). ANKTM1, a TRP-like channel expressed in nociceptive neurons, is activated by cold temperatures. Cell 112(6), 819829.
Wang, S., Dai, Y., Fukuoka, T., Yamanaka, H., Kobayashi, K., Obata, K., Cui, X., Tominaga, M., Noguchi, K. (2008). Phospholipase $\mathrm{C}$ and protein kinase A mediate bradykinin sensitization of TRPAl: A molecular mechanism of inflammatory pain. Brain 131 (Pt 5), 1241-1251.

Yang, J., Li, Y., Zuo, X., Zhen, Y., Yu, Y., Gao, L. (2008). Transient receptor potential ankyrin-1 participates in visceral hyperalgesia following experimental colitis. Neurosci Lett 440(3), 237241 .

Zimmermann, M. (1983). Ethical guidelines for investigations of experimental pain in conscious animals. Pain 16(2), 109-110. 